\title{
Discrepancies Between Predicted and Observed Intergalactic Magnetic Field Strengths from the Universe's Total Energy: Is It Contained Within Submatter Spatial Geometry?
}

\author{
Michael A. Persinger \\ Laurentian University, Sudbury, P3E 2C6, Ontario, Canada \\ E-mail address: mpersinger@laurentian.ca
}

\begin{abstract}
Although the gravitational energy within the distance of the radius of a singularity for a current estimated mass of the universe is equal to $\sim 10^{69}$ Joules, congruent solutions for different ages of the universe reflect changes by a factor of $\pi$ or $8 \pi$ for identities. The total energy value is equal to the product of primary constants $\mathrm{G} \cdot \mu \cdot \varepsilon \cdot \hbar \cdot \sigma \cdot \mathrm{c}^{2}$ (which results in power, W) when divided by the area of smallest unit of space (area of a circle with a radius of Planck's Length) and then multiplied by the universe's current surface area and age. The conspicuous discrepancies of $\sim 2 \cdot 10^{3}$ between the predicted average magnetic intensity within the universe from that total energy and contemporary measurements can be accommodated by the quantitative product of $21.3 \pi^{4}$ derived from the classic four-dimensional metric. The equivalent electric field potential divided by the predicted magnetic intensity results in a velocity that has been suggested to reflect the latency for excess correlations to occur across the universe. The most parsimonious explanation for these results is that a large component of the magnetic manifestation of energy in the universe is recondite or occluded within its submatter spatial structure and that the required cohesion or "diffusivity" throughout the volume involves the electric field component. These quantifications may facilitate understanding of Mach's principle that any part of the universe is influenced by all of its parts.
\end{abstract}

Keywords: Gravitational energy; magnetic energy; electric energy; energy equivalence; spatial geometry; "entanglement" latency; Mach's Principle

\section{INTRODUCTION}

One of the most challenging concepts in astrophysics and physical cosmology is the source of the potential coupling between essential forces applied over distance, particularly gravitational, electric and magnetic forms, with respect to the total energy within the universe. They may be related to a common source of variance according to Einstein's preference that gravitational and electromagnetic fields would not enter as logically distinct structures [1].

That the entirety of the set, the universe, should be considered reflects Mach's principle whereby intertial and centrifugal forces are a consequence of rotations and accelerations with respect to all masses within the universe. When considering the relationships between the 
forms of energies one approach is to employ the parameters for both the contemporary and final stages of the universe.

According to the Open Cold Dark Matter model [2], the boundary would be 89.2 billion years which is similar to that derived from $G \sigma$ where $G$ is the Newtonian Gravitational Constant $6.67 \cdot 10^{-11} \mathrm{~m}^{3} \cdot \mathrm{kg}^{-1} \cdot \mathrm{s}^{-2}$ and $\sigma=1.67 \cdot 10^{-27} \mathrm{~kg} \cdot \mathrm{m}^{3}$, that is about 1 proton per cubic meter. Persinger [3] suggested that the current age is about $14 \%$ of the boundary (final) age of the universe and the congruence between this proportion and the ratio of baryonic (normal) matter measured at present was not spurious. This could indicate that dark matter and dark energy are "prescient" indicators of matter and energy yet to occur. If this were valid, then the spatial (volume) and temporal (age) extent of the boundary condition would affect universal properties throughout its levels of space and time.

Discrepancies between observed and expected quantitative solutions for total universal values have been attributed to the internal geometry of space-time below the level of discourse associated with classical matter $\left(<\sim 10^{-15} \mathrm{~m}\right)$. Explanations have ranged from Riemannian structures [4] or variations of Einstein's modified field equation where values representing the structure of space-time are combined. In this paper I suggest that the discrepancy between the observed magnetic field intensity based upon contemporary measurements [5] and that predicted by the total energy [6], may be accommodated by a multiplicative aggregate of the four-dimensional geometries employed to describe closed boundaries which implicitly exhibit constant acceleration.

\section{GRAVITATIONAL ENERGY DERIVED FROM A SINGULARITY}

Intrinsic geometry is a basis for the emergence of one of the first boundary conditions considered in cosmology, the singularity. The Schwartzchild equation for a singularity, indicated by:

$$
\mathrm{R}=2 \mathrm{MG} \cdot \mathrm{c}^{-2}
$$

indicates that $r$, the radius of the singularity with a mass $(M)$ of $2.4 \cdot 10^{52} \mathrm{~kg}$, the estimated value for the universe [6], would be $3.56 \cdot 10^{25} \mathrm{~m}$. Here $G$ is the Newtonian Gravitational Constant and $\mathrm{c}$ is the velocity of light in a vacuum $\left(3 \cdot 10^{8} \mathrm{~m} \cdot \mathrm{s}^{-1}\right.$. With this value of mass, the value for the metric must be $1.78 \cdot 10^{25} \mathrm{~m}$ which is exactly $1 / 2$ of the $\mathrm{R}$ derived from Schwartzchild's singularity.

The simplest estimate of total gravitational energy is:

$$
\mathrm{E}=\mathrm{G} \cdot\left(\mathrm{kg}^{2} \mathrm{r}^{-1}\right)
$$

Assuming $2.4 \cdot 10^{52} \mathrm{~kg}$ for the estimated mass and the radius of $3.56 \cdot 10^{25} \mathrm{~m}$ the solution results in a value of $1.08 \cdot 10^{69} \mathrm{~J}$ which is approximately $1 / 2$ of the $2.2 \cdot 10^{69} \mathrm{~J}$ calculated from the energy-mass conversion derived from the current estimated mass. This value is also the solution for kinetic energy of the Hamiltonian, which represents the total energy of a system,

or

$$
\mathrm{H}=\left(\mathrm{p}^{2} 2 \mathrm{~m}^{-1}\right)+\mathrm{V}(q)
$$

where $p$ is momentum (mv) and $q$ is a representative of the coordinate. Assuming $v=c$ and the value of $2.4 \cdot 10^{52} \mathrm{~kg}$ for the mass, the total kinetic energy in a closed system universe would be $1.08 \cdot 10^{69} \mathrm{~J}$. 
On the other hand, if the current radius of the universe is $1.26 \cdot 10^{26} \mathrm{~m}$, the energy would differ $\sim 9 \%$ from a multiplicative difference of $\pi$. If the final boundary radius at 89.2 billion years is considered, $8.99 \cdot 10^{26} \mathrm{~m}$, the multiplicative operator required to obtain the estimated energy is within $0.5 \%$ of $8 \pi$, the second derivative of the surface of a sphere. This shape is consistent with the Borowskian gravitational theory [7]. These interesting solutions suggest that intrinsic features within geometric constants and ratios, reflecting rates of rates of change, may be revealing.

The containment of total energy within the geometric structure of space can be appreciated by combing the essential parameters through dimensional analysis. The "integration" of all three "force constants" through the product of G $\mu \varepsilon$, that is G, magnetic permeability $\mu\left(1.26 \cdot 10^{-6} \mathrm{~N} \cdot \mathrm{A}^{-2}\right)$, and, permittivity $\varepsilon_{0}\left(8.85 \cdot 10^{-12} \mathrm{~A}^{2} \mathrm{~s}^{2} \mathrm{~N}^{-1} \mathrm{~m}^{-2}\right)$ is $7.44 \cdot 10^{-28}$ $\mathrm{m} \cdot \mathrm{kg}^{-1}$. When multiplied by the modified (h) Planck's constant $\left(1.06 \cdot 10^{-34} \mathrm{~kg} \cdot \mathrm{m}^{2} \cdot \mathrm{s}^{-1}\right)$ the aggregate is $48.6 \cdot 10^{-62} \mathrm{~m}^{3} \cdot \mathrm{s}^{-1}$. When multiplied by the intrinsic pressure [6] of $15.03 \cdot 10^{-11}$ $\mathrm{kg} \cdot \mathrm{m}^{-1} \cdot \mathrm{s}^{-2}$ (the product of proton density and $\mathrm{c}^{2}$ ), the power $(\mathrm{W})$ is $7.4 \cdot 10^{-71} \mathrm{~W}$.

This "constant" for power when divided by the area of a circle with the smallest distance (Planck's length) as the radius $\left(8.20 \cdot 10^{-70} \mathrm{~m}^{2}\right)$ results in a power density of $9.02 \cdot 10^{-2}$ $\mathrm{W} \cdot \mathrm{m}^{-2}$. When multiplied by the current estimated surface area of the universe $\left(1.99 \cdot 10^{53} \mathrm{~m}^{2}\right)$ and the present age $\left(4.2 \cdot 10^{17} \mathrm{~s}\right)$ the total energy is $\sim 1.19 \cdot 10^{69} \mathrm{~J}$. Considering the error in the measurement of area this value converges with the kinetic energy based upon the Hamiltonian.

\section{MAGNETIC ENERGY WITHIN THE UNIVERSE}

The classic relation for stored magnetic energy is

$$
\mathrm{J}=\mathrm{B}^{2} \cdot(2 \mu)^{-1} \cdot \mathrm{m}^{3}
$$

where $\mu$ is magnetic permeability in a vacuum $\left(4 \pi \cdot 10^{-7} \mathrm{~N} \cdot \mathrm{A}^{-2}\right), B$ is the magnetic field strength and $\mathrm{m}^{3}$ is volume. The average magnetic field strength associated with the total energy within the current estimated volume is $\sim 25 \mathrm{nT}$. For the final boundary condition $\left(3.04 \cdot 10^{81} \mathrm{~m}^{3}\right)$, the estimated average value within the volume of the universe is $1.4 \mathrm{nT}$. However the current measurements of the average magnetic field within and between galaxies are between $10^{-11}$ and $10^{-12} \mathrm{~T}$ [8]. Extremely weak unamplified extragalactic fields, as inferred from cosmic microwave background radiation, suggest upper limits of approximately $10^{-13} \mathrm{~T}[5,9]$. The median for these values is more than a factor of $10^{3}$ lower than the predicted values.

Clearly there are foci of increased magnetic field intensities. For example strong, significantly tilted interstellar magnetic field strengths of $4.6 \cdot 10^{-10} \mathrm{~T}(0.46 \mathrm{nT})$ have been inferred from deflections of solar wind plasma near the heliosheath [10]. Klazes and Crutcher [11], inferring from Zeeman splitting of $\mathrm{OH}$ absorption lines, reported $3.8 \mathrm{nT}$ field strengths for NCG 2024. Depending upon perspective Zeeman detection from neutral atoms at values approaching $\sim 1 \mathrm{nT}$ may be associated with confounding technical problems that appear as magnetic field indicators.

Whether or not this limen reflects the future upper limit of the universe's magnetic field and hence displays the random variation expected within the temporal boundary condition (the final epoch) is an interesting consideration. The $10^{-9}$ to $10^{-10} \mathrm{~T}$ strengths rather than $10^{-12}$ to $10^{-13} \mathrm{~T}$ values would still be present as highly focal features of space rather than representative of the universal volume. 
This discrepancy might be accommodated by assuming that the four-dimensional features of space contain these energies and that this non-congruence reflects the narrow increment of space and time by which the current measurements are obtained. One source for that geometry could be the intrinsic metric of Kantian space for bounded but infinite geometries. The constant contained within the products of $2 \pi r, 4 \pi r^{2}, 4 / 3 \pi r^{3}$ and $2 \pi r f$ (the time component), is $21.3 \pi^{4}$ which is $2.08 \cdot 10^{3}$.

If the calculated values for the universal magnetic field strength $(25 \mathrm{nT}$ and $1.4 \mathrm{nT})$ for the current and temporal boundary values were divided by this constant contained within the four-dimensional geometry (and the dimensions that support it) the actual values would be between $12 \cdot 10^{-12}$ and $7 \cdot 10^{-13} \mathrm{~T}$, respectively. This is within the median range of the current measured values for intergalactic magnetic field strengths.

The consideration of the $21.3 \pi^{4}$ structure is based upon the equation derived by Persinger and Koren [12], who were in search of a discrete value rather than the vague abstract of "instantaneous" to describe the latency for entanglement within the universe, to satisfy the dimensional analyses,

$$
21.3 \pi^{4} \mathrm{r}^{7} \mathrm{f}=\mathrm{G}^{2} \cdot \mathrm{m}^{2} \mathrm{~d} \cdot \mathrm{t}^{3}
$$

where $G$ is the Newtonian constant, $m$ is the mass of the universe, $d$ is its width and $t$ is the duration. The solution results in a diffusivity value for which the velocity was $2.84 \cdot 10^{23} \mathrm{~m} \cdot \mathrm{s}^{-1}$. Persinger and Koren [11] considered this value to be the velocity and hence latency for "entanglement".

The assumption was this diffusivity would occur within a network of the smallest units of length which displayed a potential continuity, like a hologram, with the whole set (the universe). For the typical width of the universe the latency for excess correlation across its extent would be about $8 \mathrm{~min}$. In other words it would not be "instantaneous", although it would appear as such for current comparisons even when "perceiving" distances of separation of thousands of parsecs.

The results of this approach indicate that the total energy within the current and boundary volume of the universe maintained as magnetic energy is primarily embedded within the geometric properties of the space. Below the increments of space associated with matter $\left(<\sim 10^{-15} \mathrm{~m}\right)$ some researchers have assumed there are an additional 7 dimensions which simulate the Kaluza-Klein derivations. This is also consistent with philosophical approaches that assume levels of discourse occur at approximately levels of $10^{3}$. In this instance the difference between the smallest expected length $\left(10^{-35} \mathrm{~m}\right)$ and $10^{-15} \mathrm{~m}$ where material phenomena emerge (a difference of $10^{20}$ order of magnitudes) would be equivalent to about 7 other dimensions, for a total of 11 dimensions.

\section{ELECTRIC ENERGY WITHIN THE UNIVERSE}

Transforming the classic estimate of voltage within a containment results in:

$$
\mathrm{V}=\sqrt{ } 2 \mathrm{E} \varepsilon^{-1}
$$

where $\varepsilon$ is the permittivity of free space and $E$ is energy. Assuming $2.2 \cdot 10^{69} \mathrm{~J}$ the potential difference would be $2.24 \cdot 10^{40} \mathrm{~V}$. Distributed over the current length of $2.52 \cdot 10^{26} \mathrm{~m}$ this would be equivalent to about $0.89 \cdot 10^{14} \mathrm{~V} \cdot \mathrm{m}^{-1}$. Interesting the quotient of $\mathrm{V} \cdot \mathrm{m}^{-1}$ and $\mathrm{kg} \cdot \mathrm{A}^{-1} \cdot \mathrm{s}^{-2}$ is 
velocity. When divided by the estimated magnetic field strength for the final boundary $\left(10^{-9}\right.$ T) the velocity would be $\sim 10^{23} \mathrm{~m} \cdot \mathrm{s}^{-1}$. This result suggests that the entanglement latency may be related to the phenomena associated with electric energy or "voltage" within the volume.

Recently Chandra Das and Misra [13] derived the likely charge of a photon as being $10^{-}$ 33 of a unit charge or $10^{-52} \mathrm{~A} \cdot \mathrm{s}$. Assuming $2.4 \cdot 10^{52} \mathrm{~kg}$ for the mass of the universe and the upper limit of the mass of a photon within the visible wavelengths to be $\sim 4.2 \cdot 10^{-44} \mathrm{~kg}$ [14], the equivalent number of photons would be $0.58 \cdot 10^{99}$. If we assume the value by Chandra Das and Misra [13], the total charge associated with the numbers of photons expressed within the visible wavelength in the life time of the universe would be $0.58 \cdot 10^{47} \mathrm{~A} \cdot \mathrm{s}$.

When multiplied by the total voltage of $2.24 \cdot 10^{40} \mathrm{~V}$ this energy would be $1.3 \cdot 10^{87} \mathrm{~J}$. Although this is much larger than the $2.2 \cdot 10^{69} \mathrm{~J}$ (the usual solution) the difference is between $10^{17}$ and $10^{18}$ which (if it were seconds) is the range of the current and boundary age of the universe. This relationship, if valid, would suggest that the classic magnitude for total energy within the universe implicitly assumes a unit time, that is, $\sim 10^{69} \mathrm{~J} \cdot \mathrm{s}^{-1}$.

In other words the total energy of the temporal set is also the total energy per unit time. This value is also convergent with the value obtained by multiplying Eddington's number $\left(1.59 \cdot 10^{79}\right)$ by a fundamental quantum $\left(2 \cdot 10^{-20} \mathrm{~J}\right)$ and $2 \pi$ the frequency of the neutral hydrogen line $\left(1.42 \cdot 10^{9} \mathrm{~s}^{-1}\right)$.

\section{CONCLUSIONS}

The implicit assumption that if all forces can be related then the operator should be reflected in the premise that energy is neither created nor destroyed but changes form. For the equivalence of the total amount of mass-energy in the universe to be congruent with the average magnetic energy throughout its volume the major proportion should now be contained within geometric structure of sub-matter space. The energy within the electric field component when related to the magnetic field energy represents an intrinsic velocity by which excess correlation or entanglement between all spaces and matter within the universal volume might be related. This perspective may facilitate the understanding of Mach's principle that any part of the universe is determined or at least influenced by all of its parts [15].

\section{References}

[1] A. Einstein, The Meaning of Relativity (2n ed) Princeton, Princeton U. Press, 1945.

[2] Y. Hoffman, O. Lahav, G. Yepes, Y. Dover Journal of Cosmology and Astroparticle Physics 10 (2007) 1-16.

[3] M. A. Persinger, International Journal of Astronomy and Astrophysics 2 (2012) 125-128.

[4] J. Audretsch, Physical Review D 27 (1983) 2872-2884.

[5] A. Neronov, I. Vovk, Science 328 (2010) 73-75.

[6] M. A. Persinger, Journal of Physics, Astrophysics and Physical Cosmology 3 (2009) 1-3.

[7] T. Borowski, International Letters of Chemistry, Physics and Astronomy 11 (2013) 44-53.

[8] S. Redfield, J. Linsky, Astrophysics Journal 583 (2008) 283-314.

[9] A. H. Minter, S. R. Spangler, Astrophysics Journal 458 (1996) 194-214. 
[10] A. Opher, F. Alouani Bibi, G. Toth, J. D. Richardson, V. V. Izmodenov, T. I. Gombosi, Nature 462 (2009) 1036-1038.

[11] I. Kazes, R. M Cutcher, Astronomy and Astrophysics 164 (1986) 328-336.

[12] M. A. Persinger, S. A. Koren, The Open Astronomy Journal 6 (2013) 10-13.

[13] M. Chandra Das, R. Misra, International Journal of Astronomy and Astrophysics 2 (2012) 97-100.

[14] L-C. Tu, J. L, G. T. Gilles, Reports on Progress in Physics 68 (2005) 77-130.

[15] J. Singh, Great Ideas and Theories of Modern Cosmology, Dover, New York, 1961. 